\title{
Time Course of Processing Emotional Stimuli as a Function of Perceived Emotional Intelligence, Anxiety, and Depression
}

\author{
Joscelyn E. Fisher, Sarah M. Sass, Wendy Heller, Rebecca Levin Silton, J. Christopher Edgar, \\ Jennifer L. Stewart, and Gregory A. Miller \\ University of Illinois at Urbana-Champaign
}

\begin{abstract}
An individual's self-reported abilities to attend to, understand, and reinterpret emotional situations or events have been associated with anxiety and depression, but it is unclear how these abilities affect the processing of emotional stimuli, especially in individuals with these symptoms. The present study recorded event-related brain potentials while individuals reporting features of anxiety and depression completed an emotion-word Stroop task. Results indicated that anxious apprehension, anxious arousal, and depression were associated with self-reported emotion abilities, consistent with prior literature. In addition, lower anxious apprehension and greater reported emotional clarity were related to slower processing of negative stimuli indexed by event-related potentials (ERPs). Higher anxious arousal and reported attention to emotion were associated with ERP evidence of early attention to all stimuli regardless of emotional content. Reduced later engagement with stimuli was also associated with anxious arousal and with clarity of emotions. Depression was not differentially associated with any emotion processing stage indexed by ERPs. Research in this area may lead to the development of therapies that focus on minimization of anxiety to foster successful emotion regulation.
\end{abstract}

Keywords: anxiety, depression, perceived emotional intelligence, ERPs, emotional Stroop

Intact mechanisms for processing emotional information facilitate appropriate reactions to one's environment that allow an individual to deal effectively with pleasant and unpleasant events or situations. For example, healthy individuals preferentially process emotional stimuli compared to neutral stimuli (Herrington et al., 2005), even when presented briefly (Junghöfer, Bradley, Elbert, \& Lang, 2001; Schupp, Junghöfer, Weike, \& Hamm, 2004). The view individuals have about their own style of negotiating emotional events or situations has been called perceived emotional intelligence (PEI; Salovey, Woolery \& Mayer, 2001; Salovey,

Joscelyn E. Fisher, Sarah M. Sass, Rebecca Levin Silton, J. Christopher Edgar, Jennifer L. Stewart, and Wendy Heller, Department of Psychology, University of Illinois at Urbana-Champaign; and Gregory A. Miller, Departments of Psychology and Psychiatry and Beckman Institute Biomedical Imaging Center, University of Illinois at Urbana-Champaign.

Joscelyn E. Fisher is now at the Maryland Psychiatric Research Center, University of Maryland School of Medicine Department of Psychiatry. J. Christopher Edgar is now at the Department of Radiology, The Children's Hospital of Philadelphia. Jennifer Stewart is now at the Department of Psychology, University of Arizona. This work was supported by an NIMH Individual National Research Service Award to Joscelyn E. Fisher (F31 MH068123) and by the National Institute of Drug Abuse (R21 A14111) and the National Institute of Mental Health (P50 MH079485, R01 MH61358, T32 MH19554, T32 MH 067533). This study was submitted in partial fulfillment of dissertation requirements for Joscelyn E. Fisher. We thank Emily Cahill and Annie LaBruna Fisher for help in preparing the figures for this paper.

Correspondence concerning this article should be addressed to Joscelyn E. Fisher, Maryland Psychiatric Research Center, Functional Neuroimaging Lab, University of Maryland School of Medicine, P.O. Box 21247, Baltimore, MD 21228. E-mail: jfisher@mprc.umaryland.edu
Woolery, Stroud, \& Epel, 2002). This concept evolved from Salovey and Mayer's (1990) model of emotional intelligence, or 'the ability to monitor one's own and others' feelings and emotions, to discriminate among them, and to use this information to guide one's thinking and actions" (p. 189). ${ }^{1}$ A self-report measure (Trait Meta Mood Scale [TMMS]; Salovey, Mayer, Goldman, Turvey, \& Palfai, 1995) was developed to assess these abilities. It assesses one's self-reported ability to notice and value emotions (Attention; e.g., "I often think about my feelings."), identify and describe specific emotions (Clarity; e.g., "I am usually very clear about my feelings."), and regulate emotions (Repair; e.g., "When I become upset, I remind myself of all the pleasures in life.").

High PEI scores are generally associated with better psychological function, and there is some evidence for distinct roles of different facets of PEI. In healthy individuals, high scores on Clarity and Repair are associated with low perceptions of stress and high life satisfaction, even when adjusting for dispositional optimism or pessimism (Extremera, Durán, \& Rey, 2007). Another

\footnotetext{
${ }^{1}$ This definition was later refined as the ability to (a) perceive emotion, (b) use emotion to facilitate thought, (c) understand emotions, and (d) manage emotion (Mayer \& Salovey, 1997). However, Salovey et al. (1995) specifically stated that the TMMS is not an emotional intelligence test but that it may help "identify core individual differences that may characterize emotionally intelligent individuals capable of disclosing their feelings to themselves and other people (p. 127)." Furthermore, the TMMS is a self-report measure and thus inherently limited as an assessment of individual differences. In fact, Salovey et al. (2001) stated that "it will be difficult to continue conceptualizing emotional intelligence as a kind of intelligence ... if the field continues to rely on self-report instruments as the way to assess it" (p. 301). In the present paper, what this scale measures is referred to as PEI (Salovey et al., 2001; Salovey et al., 2002).
} 
study indicated that low perception of stress was observed only for individuals with high scores on Attention, Clarity, and a measure of intensity of emotion (Gohm, Corser, \& Dalsky, 2005). In women, high Clarity was associated with less depression and fatigue after an acute stressor, whereas high Repair was associated with less depression and anger one day after exposure to an acute stressor (Ramos, Fernandez-Berrocal, \& Extremera, 2007).

As high PEI is associated with psychological well-being, low scores on these dimensions might be associated with psychopathology, especially as anxiety and depression are characterized by emotional disturbances (Berenbaum, Raghavan, Le, Vernon, \& Gomez, 2003). For example, individuals who experienced panic attacks reported greater emotional avoidance, difficulty accepting emotions, and lower emotional clarity than did individuals without a history of panic attacks (Tull \& Roemer, 2007). Similarly, worry and generalized anxiety disorder (GAD) have been associated with deficits in emotional clarity, acceptance of emotions, ability to engage in goal-directed behaviors when distressed, impulse control, and access to effective regulation strategies (SaltersPedneault, Roemer, Tull, Rucker, \& Mennin, 2006).

When using the TMMS to characterize PEI, depressed individuals scored lower on Attention (Rude \& McCarthy, 2003) and Clarity (Fernández-Berrocal, Alcaide, Extremera, \& Pizarro, 2006; Rude \& McCarthy, 2003) than did nondepressed individuals. Clarity and Repair were also negatively associated with anxiety (Fernández-Berrocal et al., 2006). However, elevated TMMS scores also have been associated with psychopathology. Individuals prone to worry but having low levels of depression reported high Attention and Clarity, whereas those with low worry and high depression reported low Attention and Clarity (Bredemeier, Berenbaum, Boden, \& Thompson, 2007). Finally, individuals high in both worry and depression had slightly elevated Attention and low Clarity (Bredemeier et al., 2007). These findings indicate complex relationships between facets of PEI and psychopathology.

Underspecification of diagnoses or symptoms may contribute to inconsistent findings regarding psychopathology and PEI. For instance, assessing both anxiety and depression is important, given high comorbidity and their differential behavioral and neural relationships to emotion (e.g., Keller et al., 2000). Rude and McCarthy (2003) examined depression but did not assess anxiety, which may have influenced the findings. Other studies did not distinguish between types of anxiety (e.g., Fernandez-Berrocal et al., 2006). This differentiation is important, as studies have distinguished two dimensions of anxiety: anxious apprehension (characterized by worry) and anxious arousal (characterized by physical or somatic concerns) that differ according to patterns of lateralized brain activity measured via functional MRI (fMRI) or electroencephalography (EEG; e.g., Engels et al., 2007; Nitschke, Heller, Imig, McDonald, \& Miller, 2001; Nitschke, Heller, Palmieri, \& Miller, 1999). Anxious apprehension has been associated with left-frontal specialization for verbal iterative processes (Engels et al., 2007; Heller, Nitschke, Etienne, \& Miller, 1997). In contrast, anxious arousal is associated with right posterior areas (Engels et al., 2007; Heller et al., 1997; Keller et al., 2000) that are components of a vigilance network (Herrington et al., 2005; Nitschke et al., 1999, 2000). Furthermore, recent fMRI findings indicate a different pattern of activity associated with depression. Herrington et al. (in press) found that depressed and nondepressed groups showed a leftward lateralization of dorsolateral prefrontal cortex
(DLPFC) activity for positive stimuli. The depressed group also demonstrated a pattern of less left and more right lateralized DLPFC activity to negative versus neutral stimuli, consistent with previous resting EEG studies (for reviews, see Davidson, Pizzagalli, Nitschke, \& Putnam, 2002; Heller \& Nitschke, 1997). These distinct mechanisms of stimulus processing associated with each type of anxiety and depression are confounded when anxiety is studied as a uniform construct, and when anxiety is not separated from effects of depression.

It is unknown how PEI affects emotional stimulus processing, but it is well established that individuals who are anxious or depressed tend to display abnormalities in emotion processing. Anxiety has often been associated with abnormalities in various stages of processing negative stimuli, although a consistent picture has not yet emerged from the literature. Anxious individuals direct attention toward threat during early stages of processing and direct attention away during later stages (Pflugshaupt et al., 2005; Rohner, 2002). Yet there is also evidence that anxious individuals maintain attention to and have difficulty disengaging from threat (Fox, Russo, Bowles \& Dutton, 2001; Yiend \& Mathews, 2001). Some studies suggested that anxiety facilitates perception of negative stimuli (Öhman, Flykt, \& Esteves, 2001), though others claimed that detection of threat is not faster in anxiety (Bar-Haim, Lamy, Pergamin, Bakermans-Kranenburg, \& van Ijzendoorn, 2007; Rinck, Reinecke, Ellwart, Heuer, \& Becker, 2005). In contrast, individuals who are depressed have been reported either to attend to negative and positive stimuli equally (McCabe \& Gotlib, 1995 ) or to be less responsive to positive stimuli (Berenbaum \& Oltmanns, 1992; Dunn, Dalgleish, Lawrence, Cusack, \& Ogilvie, 2004). Because of these differences between anxiety and depression, PEI may interact with each of them in unique ways to affect emotion processing.

The distinct contribution of PEI alone and in combination with anxiety and depression to the processing of emotional stimuli remains to be determined. To investigate this issue, the present study used the emotion-word Stroop (ES) task. The participant is asked to ignore the meaning of a word to respond to its color. To the extent that processing resources are devoted to the taskirrelevant emotional meaning of a stimulus, an interference effect can occur, manifested in increased reaction time. Robust behavioral interference effects to threat-related words have been demonstrated in this task (for a review, see Williams, Mathews, \& MacLeod, 1996), especially in samples with anxiety (e.g., Egloff \& Hock, 2003; Fox, 1993; Mathews \& MacLeod, 1985) and depression $^{2}$ (Lim \& Kim, 2005; for a review, see Williams et al., 1996). Performance on this task is also associated with differences in TMMS scores: Individuals scoring high on the Attention subscale were more attentive to both positive and negative words in the ES task, as indicated by longer reaction times (Coffey, Berenbaum, \& Kerns, 2003). Thus, the ES task is a promising tool for investigating the intersection of psychopathology and PEI.

\footnotetext{
${ }^{2}$ Comorbid anxiety may have played a role in these results. These studies either did not assess anxiety (e.g., Gotlib \& Cane, 1987) or used measures that do not differentiate between depression and anxiety (Lim \& Kim, 2005; for review of these measures, see Nitschke et al., 2001). Williams and Nulty (1986) recruited "worriers" but assessed depression, likely leading to a sample with comorbid anxiety and depression.
} 
Continuous temporal dynamics of stimulus processing during the ES task have been characterized by ERPs (W. Li, Zinbarg, \& Paller, 2007; McNeely, Lau, Christensen, \& Alain, 2008; Metzger \& Orr, 1997; Pérez-Edgar \& Fox, 2003; Sass et al., in press; Thomas, Johnstone, \& Gonsalvez, 2007; van Hooff, Dietz, Sharma, \& Bowman, 2008). For instance, Sass et al. (in press) found that emotionally arousing words elicited an enhanced P100 component in an anxious arousal group, indicating early preferential processing of emotionally arousing stimuli. Both P100 and P200 have been associated with early attention to emotional stimuli (e.g., Pourtois, Grandjean, Sander, \& Vuilleumeir, 2004; Thomas et al., 2007). Later processing can be measured by P300, a component that can increase with task difficulty and is generally interpreted as an index of the cognitive resources allocated to a task (Donchin \& Coles, 1988; Yee \& Miller, 1994). Negative stimuli have been associated with larger P300, interpreted as allocation of additional resources for categorization of stimuli (W. Li et al., 2007; Thomas et al., 2007). P300 latency is interpreted to reflect stimulus evaluation time (Donchin \& Coles, 1988). Metzger and Orr (1997) reported a trend for later P300 latency to traumarelated words in patients with posttraumatic stress disorder (PTSD), indicating delayed evaluation of such words. A subsequent slow-wave component (600-1,000 ms) was larger to negative words (Pérez-Edgar \& Fox, 2003), which may reflect extended processing of task-relevant (color) information to counter the processing of the most difficult task-irrelevant (word meaning) information (West \& Alain, 2000).

Several of the ERP components described above can be used to characterize the role of psychopathology and PEI in the processing of emotional stimuli. It is likely that anxious arousal will be associated with P200, as anxiety disorders characterized by arousal (e.g., panic disorder) are associated with earlier preferential attention (larger amplitude and shorter latency of P200; Hanatani et al., 2005; Pauli, Amrhein, Mühlberger, Dengler, \& Wiedemann, 2005; Yee \& Miller, 1988). As patients with panic disorder did not differentiate neutral stimuli from emotional stimuli until after 700 $\mathrm{ms}$ during the recognition portion of an emotional memory paradigm (Windmann, Sakhavat, \& Kutas, 2002), P200 amplitude may not differentiate emotionally arousing from neutral stimuli in the anxious arousal group during the ES task. High Attention to emotion may also be associated with increased P200 amplitude, as focusing on one's emotions may prime the individual to perceive emotional stimuli earlier than those with low Attention to emotion.

On the other hand, there is mixed evidence for an association between trait anxiety or disorders characterized by elevated apprehension (e.g., generalized anxiety disorder) and enhanced early sensory processing of emotional stimuli, with some studies finding evidence for it (e.g., Sass et al., in press) and others studies either not finding evidence (Drake, Pakalnis, Phillips, Pamadan, \& Hietter, 1991; Turan et al., 2002) or finding a combination of early and later preferential processing of emotional stimuli (e.g., W. Li et al., 2007). For example, $\mathrm{P} 100$ is modulated by emotional stimuli (Fox, Derakshan, \& Shoker, 2008; Holmes, Nielsen, \& Green, 2008; X. Li, Li, \& Luo, 2005), but evidence for its association with trait anxiety is mixed. Some studies observed larger amplitude in individuals with high anxiety (e.g., Holmes et al., 2008; X. Li et al., 2005), whereas others found no effect (e.g., Fox et al., 2008). Thus, it is unclear whether P100 would be associated with anxious apprehension. As anxious apprehension involves worry about neg- ative outcomes that may occur, it was predicted that in the present study anxious apprehension would be associated with increased evaluation of negative stimuli (delayed P300 latency), especially in combination with Clarity of emotion, as this facet of PEI would likely require more time to process or categorize an event or stimulus.

Slow wave would provide information about late processing associated with anxiety, depression, and PEI. Both depression and anxious arousal have been associated with slow wave in paradigms with emotional stimuli. Patients with panic disorder (who are generally concerned about physical sensations) had larger slow wave to body-related words than did controls, reflecting the fact that these words were more meaningfully encoded in this group (Pauli et al., 1997). Depression has been associated with smaller slow-wave amplitude to positive stimuli than to negative and neutral stimuli (Shestyuk, Deldin, Brand, \& Deveney, 2005) and equivalent slow wave responses to both positive and negative stimuli (Deveney \& Deldin, 2004), suggesting that depressed individuals do not engage in elaborative processing of positive stimuli and do not avoid extended processing of negative stimuli as healthy individuals do. In the present study, it was predicted that depression would be associated with equivalent slow wave to positive and negative stimuli, whereas anxious arousal would be associated with increased slow wave to negative stimuli. ${ }^{3}$ As ERPs reflecting late processing have been interpreted as reflecting controlled strategies in patients with panic disorder (Windmann et al., 2002), and emotion Repair involves reinterpreting a negative situation in a positive light, emotion Repair should also be associated with increased slow wave, especially to negative stimuli. In addition, Attention to emotion and Clarity of emotion may also be associated with enhanced slow wave to positive and negative stimuli, as the propensity to "pay attention to," or be "very clear about my" feelings may be facilitated by elaborative processing.

The present study assessed distinct aspects of anxiety and depression with the goal of determining whether and how facets of PEI in combination with these psychopathology dimensions are differentially associated with processing emotional stimuli. These relationships were investigated in a large sample of nonclinical individuals selected to have a range of scores on psychopathology measures, to study these characteristics from a dimensional perspective and avoid confounding effects of treatment and chronicity (Fernandes \& Miller, 1995). As outlined in Table 1, it was hypothesized that P100 would be sensitive to emotional stimuli, but it was unclear whether it would be sensitive to anxiety (or PEI) given the mixed literature reviewed above. Attention to emotion and anxious arousal would each be associated with heightened early perception evidenced by enhanced P200 amplitude to all stimuli (positive, neutral, and negative) and Clarity of emotion and anxious apprehension would be associated with increased time to

\footnotetext{
${ }^{3}$ Sass et al. (in press) hypothesized that anxious individuals would preferentially process emotionally arousing words early, in combination with later avoidance reflected in components after $300 \mathrm{~ms}$. However, that study did not investigate components after $580 \mathrm{~ms}$. Therefore, the present study also investigated a later slow wave to determine whether emotionally arousing words are differentiated by levels of anxiety later in the processing stream.
} 
Table 1

Hypothesized Associations Between Constructs

\begin{tabular}{|c|c|c|c|c|c|c|c|c|c|c|c|}
\hline \multicolumn{3}{|c|}{ P100 amplitude } & \multicolumn{3}{|c|}{ P200 amplitude } & \multicolumn{3}{|c|}{ P300 latency } & \multicolumn{3}{|c|}{ Slow-wave amplitude } \\
\hline Positive & Neutral & Negative & Positive & Neutral & Negative & Positive & Neutral & Negative & Positive & Neutral & Negative \\
\hline $\begin{array}{l}\text { Increased } \\
\text { compared to } \\
\text { neutral }\end{array}$ & & $\begin{array}{l}\text { Increased } \\
\text { compared to } \\
\text { neutral }\end{array}$ & $\begin{array}{c}\text { Anx arou, } \\
\text { AE }\end{array}$ & $\begin{array}{c}\text { Anx arou, } \\
\mathrm{AE}\end{array}$ & $\begin{array}{c}\text { Anx arou, } \\
\text { AE }\end{array}$ & $\begin{array}{l}\text { Anx app, } \\
\text { CE }\end{array}$ & $\begin{array}{l}\text { Anx app, } \\
\text { CE }\end{array}$ & $\begin{array}{l}\text { Anx app, } \\
\text { CE }\end{array}$ & $\begin{array}{c}\text { Dep, } \\
\text { AE }\end{array}$ & & $\begin{array}{c}\text { Dep, } \\
\text { Anx arou, } \\
\text { ER, AE }\end{array}$ \\
\hline
\end{tabular}

Note. $\quad$ Anx app = anxious apprehension; Anx arou = anxious arousal; Dep $=$ depression; $\mathrm{AE}=\mathrm{Attention}$ to emotion; CE $=\mathrm{Clarity}$ of emotion; ER $=$ Emotion repair.

categorize stimuli (indexed by P300 latency). Both emotion Repair and Attention to emotion, in addition to anxious arousal and depression, would be associated with extended processing, but in different emotion conditions. Depression would be associated with equivalent slow wave to positive and negative stimuli, whereas arousal would be associated with enhanced slow wave to negative stimuli. Emotion Repair and Attention to emotion would be associated with enhanced slow wave, though emotion Repair would be associated with negative stimuli and Attention to emotion would be associated with both positive and negative stimuli.

\section{Method}

\section{Participants}

Over 1,000 participants in undergraduate psychology classes across several semesters filled out the Penn State Worry Questionnaire (PSWQ; Meyer, Miller, Metzger, \& Borkovec, 1990; Molina $\&$ Borkovec, 1994) and the anxious arousal and anhedonic depression scales of the Mood and Anxiety Symptom Questionnaire (MASQ; Watson, Clark, et al., 1995; Watson, Weber, et al., 1995). Five groups were recruited for a larger fMRI and EEG study based on combinations of scores from three scales: the PSWQ, the MASQ Anxious Arousal scale, and an eight-item subscale of the MASQ Anhedonic Depression scale that emphasizes depressed mood rather than low positive affect (Nitschke et al., 2001). Those who had scores at the 80th percentile or higher on one scale and at the 50th percentile or lower on the other two scales were recruited for three high-scoring groups: high anxious apprehension only, high anxious arousal only, or high anhedonic depression only. The comorbid group had scores at the 80th percentile or higher on all three scales, and the healthy control group had scores in the 50th percentile or lower on all three questionnaires. The comorbid group was included as a second control group, as it most closely resembles the majority of studies in the anxiety literature that have relied on the State Trait Anxiety Inventory (STAI; Spielberger, Gorsuch, \& Lushene, 1970), a measure which is conflated with depression and likely indexes negative affect more broadly (Nitschke et al., 2001). All participants were right-handed, native speakers of English with self-reported normal color vision. Participants were given a laboratory tour, informed of the procedures of the study, and screened for claustrophobia or contraindications for MRI participation.

Present analyses are based on 88 paid participants: 18 comorbid, 14 anxious apprehension, 14 anxious arousal, 15 anhedonic depression, and 27 control. ${ }^{4}$ Scores on the TMMS (Salovey et al.,
1995) were obtained for 62 of the participants. Participants were 18 to 34 years old $(M=19.0, S D=1.8), 53 \%$ women, $84 \%$ White, medically healthy by self-report and right-handed as determined by the Edinburgh Handedness Inventory (Oldfield, 1971). Following the group questionnaire screening session, individuals invited for participation attended a lab tour, an EEG session, an fMRI session, and a Structured Clinical Interview for DSM-IV Axis I Disorders (SCID; First, Spitzer, Gibbon, \& Williams, 1997). Participants completed the emotion-word and color-word Stroop tasks, while 3T fMRI data and again while 64-channel EEG data were collected. The order of presentation of the two Stroop tasks within a session was counterbalanced across participants, as was the order of the fMRI and EEG sessions, with the SCID session usually in-between. Data from the emotion-word Stroop task during the EEG session were considered in the present study.

\section{Measures}

During the initial lab tour, participants were administered the TMMS and re-administered the PSWQ and the MASQ. Present analyses are based on these scores because they were obtained closer in time to the EEG measurements. The test-retest reliabilities were: PSWQ, $r(81)=.91, p<.001$; MASQ Anxious Arousal, $r(84)=.71, p<.001 ;$ and MASQ Anhedonic Depression 8-item subscale, $r(84)=.64, p<.001$. The TMMS has adequate internal consistency among the three scales (Attention: $\alpha=.86$; Clarity: $\alpha=.87$; Repair: $\alpha=.82$ ) and convergent validity when compared to measures of related constructs (Salovey et al., 1995).

\section{Task}

An effective design for the emotion-word Stroop task uses blocked stimuli (Bar-Haim et al., 2007; Compton, Heller, Banich, Palmieri, \& Miller, 2000). The task was implemented as blocks of positive or negative emotion words alternating with blocks of neutral words. Participants received 256 trials in 16 blocks (four positive, eight neutral, four negative) of 16 trials, with a variable intertrial interval (ITI) 2,000 $\pm 225 \mathrm{~ms}$ between trial onsets. A trial began with the presentation of a word for $1500 \mathrm{~ms}$, followed by a

\footnotetext{
${ }^{4}$ Of the present sample, 38 of the 88 participants were included in Sass et al. (in press), which was confined to anxious apprehension, anxious arousal, and control groups. The present sample added comorbid and depressed groups, investigated slow-wave ERP scores, and evaluated the three facets of the PEI via TMMS scores.
} 
fixation cross for 275 to $725 \mathrm{~ms}$. Each trial consisted of one word presented in one of four colors (red, yellow, green, blue) on a black background, with each color occurring equally often within word type (positive, neutral, negative). Each participant received one of eight orders designed to minimize stimulus order effects. In four of the eight presentation orders, the first and third blocks were neutral words, with positive and negative blocks second or fourth and valence order counterbalanced across participants. The remaining four presentation orders complemented these, with the first and third blocks consisting of positive or negative words and the second and fourth consisting of neutral words.

Emotional and neutral words preceded each other equally often, and no word was repeated throughout the experiment. Within a block, each color appeared four times, and trials were pseudorandomized such that no more than two trials featuring the same color appeared in a row. After every fourth block, there was a brief rest period. In addition to the 16 word blocks, there were four fixation blocks - one at the beginning, one at the end and two in the middle of the session. In the fixation condition, instead of a word, a brighter fixation cross was presented for $1,500 \mathrm{~ms}$.

The 256 word stimuli were selected from the Affective Norms for English Words (ANEW) set (Bradley \& Lang, 1999). Sixtyfour were positive (e.g., birthday, ecstasy, laughter), 64 were negative (e.g., suicide, war, victim), and two sets of 64 were neutral (e.g., hydrant, moment, carpet). The words were selected on the basis of established norms for valence, arousal, and frequency of usage in the English language (Bradley \& Lang, 1999; Toglia \& Battig, 1978) and ranged from three to eight letters in length. Words were presented in capital letters using Tahoma 72-point font at a distance of $1.35 \mathrm{~m}$ from the participant's eyes, for a vertical span of 1.5 degrees and a horizontal span of 2.5 degrees to 9.3 degrees. Instructions were read verbatim by experimenters to assure that participants understood task requirements. The participant performed 32 practice trials before the actual tasks began. No participants failed to understand the task instructions or the mapping between colors and buttons after completing practice trials. Participants responded with the middle and index fingers of each hand using a four-button response box.

\section{Electrophysiological Recording}

Participants were seated in a comfortable chair in a quiet room connected to the adjacent equipment room by intercom. EEG was recorded with a custom-designed Falk Minow (Munich, Germany) 64-channel cap with $\mathrm{Ag} / \mathrm{AgCl}$ EEG electrodes spaced equidistantly. The left mastoid served as the reference during recording. By placing electrodes above and below each eye and near the outer canthus of each eye, vertical and horizontal EOG were recorded. Electrode impedances were maintained below 20 Kohms. Amplifier bandpass was 0.1 to $100 \mathrm{~Hz}$, with digitization at $250 \mathrm{~Hz}$.

\section{Data Reduction}

Artifacts were removed and eyeblinks corrected with Brain Electrical Source Analysis (BESA v. 5.1.8) software (Berg \& Scherg, 1994). Trials were rejected if reaction time (RT) was not between 200 and $1,000 \mathrm{~ms}$, as responses less than $200 \mathrm{~ms}$ would not be credible as genuine choices made after stimulus onset and responses greater than $1,000 \mathrm{~ms}$ likely reflect trials in which the participant was not engaged in the task. The percentage of excluded trials (including trials in which the participant did not respond) was low (1.4\%). Mean RT across all trials and participants was $633 \mathrm{~ms}$ (SD $97 \mathrm{~ms}$ ). For each participant, all trials for each emotion word type were averaged because the error rate was low (4.5\%, SD 3.9\%), and the phenomena of interest were not expected to vary according to error rates. The electrode configuration was transformed to a standardized 81-channel montage placed according to the 10-10 system provided in BESA (Perrin, Pernier, Bertrand, \& Echallier, 1989). An average reference was computed for each time point as the mean voltage over the interpolated amplitudes of the 81 standard virtual scalp electrodes. Data were exported from BESA and baseline adjusted by subtracting the average amplitude for the $200 \mathrm{~ms}$ before stimulus onset. Waveform averages were smoothed using a 101-weight, 0.1 to $10 \mathrm{~Hz}$ digital filter (Cook \& Miller, 1992; Nitschke, Miller, \& Cook, 1998). Amplitude and latency scores were obtained for each of the 81 channels.

For each participant, peak amplitudes were calculated within the following latency windows for each electrode in a specific scalp region (see Figure 1): P100 (88-148 ms), P200 (148-248 ms) and P300 (348-768 ms). Mean amplitude was calculated for slow wave (780-1,200 ms). Groups of four adjacent electrodes were selected to create two composite region scores for each hemisphere to obtain a more stable measure of regional activity. For each window, a score was calculated for each hemisphere in a region by averaging the scores of the individual electrodes in the hemisphere (see Figure 2), thus creating four scores (frontal left: AF3, F1, F3, F5; frontal right: AF4, F2, F4, F6; parietal left: P1, P3, P5, PO3; parietal right: $\mathrm{P} 2, \mathrm{P} 4, \mathrm{P} 6, \mathrm{PO} 4)$.

\section{Data Analysis}

As P100 is maximal over occipitoparietal sites (e.g., Fox et al., 2008), P100 was scored over the parietal region. P200 scores over frontal sites were chosen, as maximal effects were observed there in this dataset and in prior literature (Luck, 2005; Pauli et al., 2005). Parietal regions were chosen for P300 (Luck, 2005) and slow wave, as several studies have reported a slow wave over parietal regions in Stroop paradigms (Ilan \& Polich, 1999; PerezEdgar \& Fox, 2003) and in other emotional processing paradigms (Keil et al., 2002; Shestyuk et al., 2005).

A Group (comorbid, anxious apprehension, anxious arousal, anhedonic depression, control) $\times$ Emotion (positive, neutral, negative) $\times$ Hemisphere (left, right) multivariate analysis of variance (MANOVA) including linear and quadratic trends was conducted separately for P100 amplitude, P200 amplitude, P300 amplitude and latency, and slow wave amplitude scores. Hemisphere was included as an exploratory variable because lateralized ERPs have not been a focus in prior literature. Group effects were followed up with LSD pairwise comparisons.

Hypotheses guided hierarchical linear regressions used to investigate the relationship of anxiety and TMMS to emotion processing stages. PSWQ, MASQ, and TMMS scores were used to predict P200 amplitude, P300 amplitude and latency, and slow wave across all participants. 

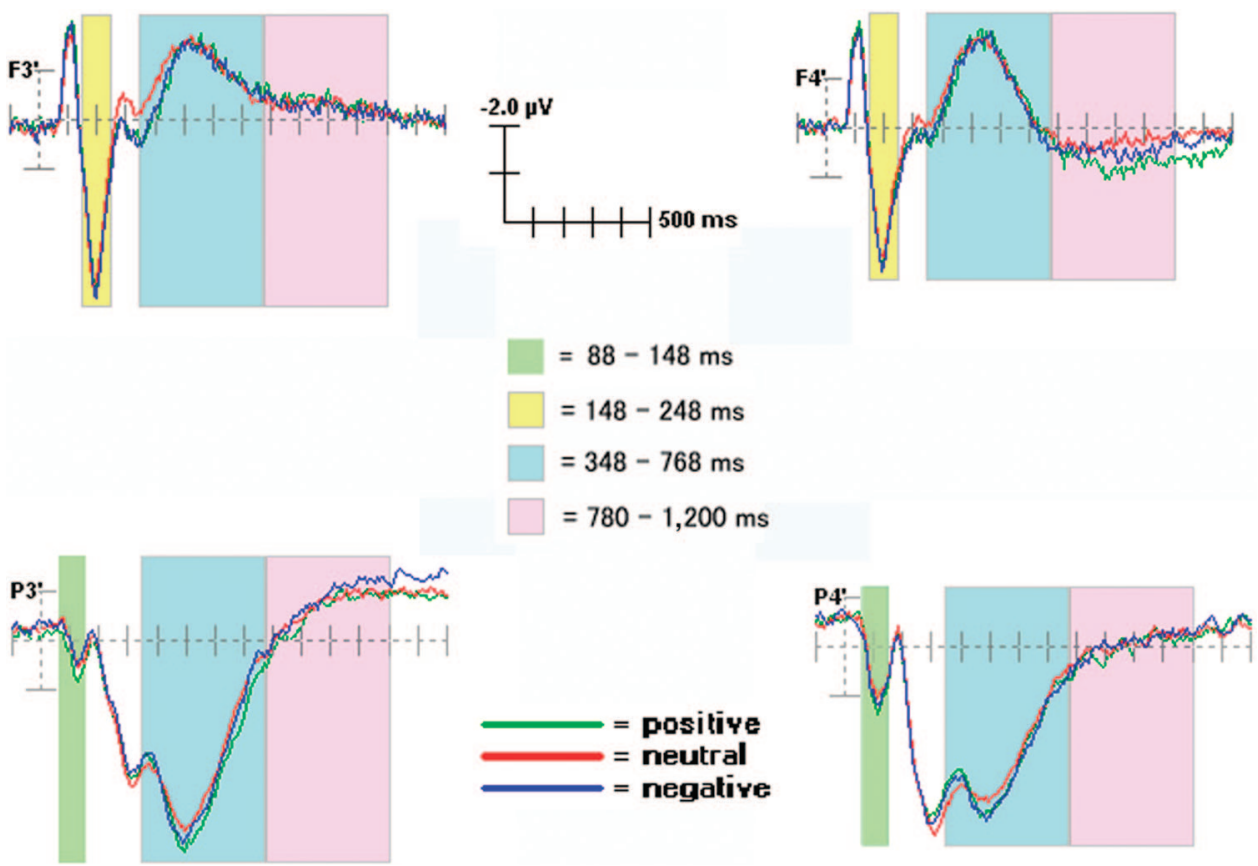

Figure 1. Event-related potential (ERP) scoring windows illustrated for representative channels (F3, F4, P3 and P4). All channels referred to an average reference. Waveforms from $100 \mathrm{~ms}$ prior to stimulus onset to $1,400 \mathrm{~ms}$ after stimulus onset. Each tick mark on the $x$-axis represents $100 \mathrm{~ms}$.

\section{Results}

\section{Psychopathology Measures and Behavioral Performance}

Consistent with the literature, anxious apprehension, anxious arousal, and anhedonic depression were correlated with Clarity and Repair across all participants (see Table 2). To determine which of the three psychopathology characteristics accounted for unique variance in Clarity of emotion scores, hierarchical regressions were run with anxious apprehension, anxious arousal, and anhedonic depression as predictors. The overall model accounted for $28 \%$ of the variance in Clarity of emotion, $F(3,58)=7.49, p<$ .001 . Only anxious arousal accounted for unique variance when added last to the three-predictor model, $\Delta R^{2}=.24, t(58)=-3.54$, $p=.001$. The same predictors accounted for $23 \%$ of the variance in emotion Repair, $F(3,58)=5.76, p=.002$, with anhedonic depression accounting for unique variance when added last, $\Delta R^{2}=$ $.19, t(58)=-2.57, p=.013$. The predictors did not contribute to the Attention to emotion facet of PEI.

RT was similar across the three conditions (positive: $634 \mathrm{~ms}, S D$ $95 \mathrm{~ms}$; neutral: $631 \mathrm{~ms}, S D 96 \mathrm{~ms}$; negative: $633 \mathrm{~ms}, S D 101 \mathrm{~ms}$ ). There were no group differences in RT or error rate in any condition, positive: $F(4,80)=.94, p=.45$; neutral: $F(4,80)=$ $1.81, p=.14$; negative: $F(4,80)=1.0, p=.41$, and RT and error rates were not correlated with any of the psychopathology or PEI measures. There was a trend for Attention to emotion to be negatively correlated with RT in neutral and negative conditions, neutral: $r(60)=-.24, p=.06$; negative: $r(60)=-.22, p=.09$, which is somewhat consistent with a prior emotion-word Stroop study (Coffey et al., 2003).

\section{Event-Related Potential Analyses}

P100. At posterior sites, P100 amplitude was larger over the right hemisphere than the left hemisphere, $F(1,83)=30.63, p<$ .001 , and was marginally larger to positive and negative than to neutral stimuli, reflected in the quadratic Emotion effect, $F(1$, $83)=2.71, p=.10$. There were no correlations between P100 and measures of psychopathology or PEI scores.

P200. P200 amplitude was larger over the left than the right hemisphere, $F(1,83)=13.77, p<.001$. There were no group differences and no main effects or interactions of emotion or hemisphere with group.

As predicted, Attention to emotion was positively correlated with P200. However, this was not limited to with emotionally arousing stimuli, as expected. Instead, Attention to emotion was correlated with P200 for all stimuli measured over either hemisphere, with higher scores associated with larger amplitude (not significant for negative stimuli over the right hemisphere; see Table 3 for correlations).

Anxious arousal and Attention to emotion were included as predictors in a regression to evaluate the hypothesis that each is a unique predictor of P200. Table 4 shows that anxious arousal and Attention to emotion each accounted for unique variance in P200 amplitude in each stimulus condition when added last to the two-predictor model (anxious arousal was significant in models predicting P200 to all stimuli over the right frontal region, but only negative stimuli over the left frontal region).

P300. There was a linear effect of emotion, $F(1,83)=4.80$, $p=.03$, on $\mathrm{P} 300$ latency, reflecting later evaluation of positive stimuli. A quadratic Emotion $\times$ Hemisphere interaction, $F(1$, $83)=4.54, p=.04$, indicated that $\mathrm{P} 300$ latency for neutral stimuli 


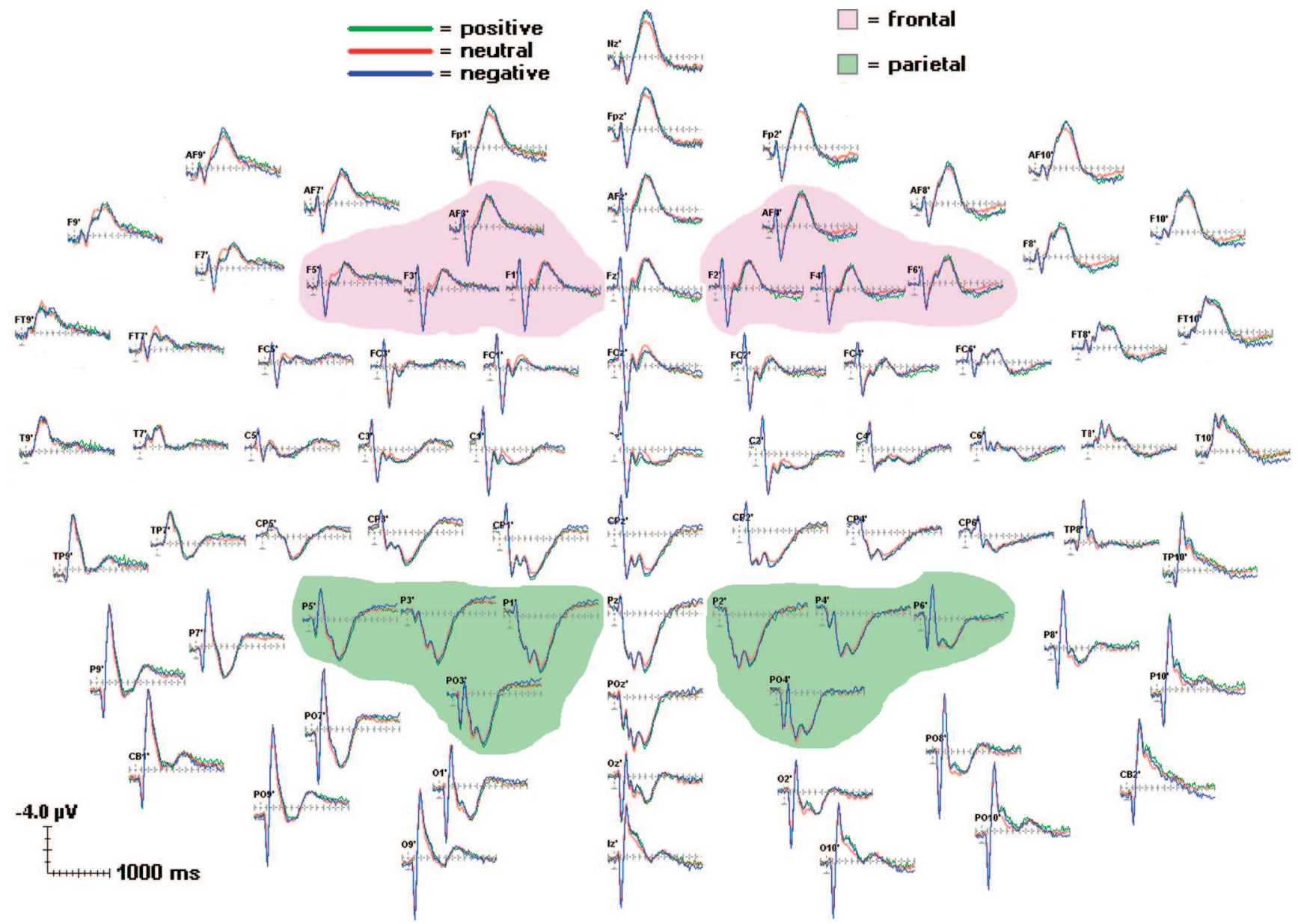

Figure 2. Grand average $(n=88)$ for each emotion condition showing scalp topography for individual channels and regional groupings of channels. All channels referred to an average reference. Waveforms from 100 $\mathrm{ms}$ prior to stimulus onset to $1,400 \mathrm{~ms}$ after stimulus onset. Each tick mark on the $x$-axis represents $100 \mathrm{~ms}$.

occurred later over the left hemisphere $(p=.02)$. The main effect of group was not significant, $F(4,83)=1.85, p=.13$.

To address the hypothesis that anxious apprehension and Clarity of emotion would be associated with evaluation time during negative stimuli, anxious apprehension and Clarity of emotion were entered as predictors of P300 latency to negative stimuli in a hierarchical regression (see Table 4). The overall model accounted for $12 \%$ of the variance of P300 latency to negative stimuli over the right posterior region ( $p=.03$ ). When added last, neither anxious apprehension nor Clarity of emotion added significant variance $(p s>.05)$. The overall models that predicted P300 latency to positive and neutral stimuli (calculated as comparisons) were not significant $(p s>.05)$.

To determine whether this finding was specific to negative stimuli, another series of hierarchical regressions was done. Both P300 latency to positive stimuli and P300 latency to neutral stimuli were entered in the first step as predictors of P300 latency to negative stimuli. Clarity of emotion and anxious apprehension were added either second or third. Clarity of emotion did not add significant variance with or without anxious apprehension in-

Table 2

Correlations Between Psychopathology and Emotion Regulation Measures

\begin{tabular}{|c|c|c|c|c|c|c|}
\hline \multirow[b]{2}{*}{ Measure } & \multicolumn{2}{|c|}{ Attention to emotion } & \multicolumn{2}{|c|}{ Clarity of emotion } & \multicolumn{2}{|c|}{ Emotion repair } \\
\hline & $r$ & $p$ & $r$ & $p$ & $r$ & $p$ \\
\hline Anxious apprehension & .09 & .468 & -.27 & .035 & -.23 & .078 \\
\hline Anxious arousal & -.19 & .144 & -.50 & $<.001$ & -.35 & .005 \\
\hline Anhedonic depression & -.24 & .058 & -.30 & .019 & -.43 & .001 \\
\hline
\end{tabular}

Note. $\quad N=62$. 
Table 3

Correlations Between Attention to Emotion and P200 by Emotion Condition and Hemisphere

\begin{tabular}{|c|c|c|c|c|c|c|c|c|c|c|c|c|}
\hline \multirow[b]{3}{*}{ Variable } & \multicolumn{6}{|c|}{ LH } & \multicolumn{6}{|c|}{$\mathrm{RH}$} \\
\hline & \multicolumn{2}{|c|}{ Positive } & \multicolumn{2}{|c|}{ Neutral } & \multicolumn{2}{|c|}{ Negative } & \multicolumn{2}{|c|}{ Positive } & \multicolumn{2}{|c|}{ Neutral } & \multicolumn{2}{|c|}{ Negative } \\
\hline & $r$ & $p$ & $r$ & $p$ & $r$ & $p$ & $r$ & $p$ & $r$ & $p$ & $r$ & $p$ \\
\hline Attention to emotion & .34 & .01 & .27 & .04 & .28 & .03 & .28 & .03 & .26 & .04 & .19 & .14 \\
\hline
\end{tabular}

Note. $\quad N=62 . \mathrm{LH}=$ left hemisphere; $\mathrm{RH}=$ right hemisphere.

cluded. In contrast, anxious apprehension marginally contributed whether Clarity of emotion was already included, $\Delta R^{2}=.024, t=$ $-1.75, p=.085$, or not, $\Delta R^{2}=.027, t=-1.86, p=.068$.

Predictions were about P300 latency, but P300 amplitude was also analyzed for comparison with prior literature about P300 amplitude and emotionally arousing stimuli. There was a trend for larger P300 amplitude to both positive and negative stimuli than P300 to neutral stimuli at left-hemisphere sites, reflected in the quadratic Emotion $\times$ Hemisphere effect, $F(1,83)=3.38, p=.07$, which is consistent with literature indicating P300 sensitivity to emotionally arousing stimuli.

Slow wave. Slow-wave amplitude was larger (more negative going) over the left than right hemisphere, $F(1,82)=33.6, p<$ .001 . It was hypothesized that the three PEI subscales, in addition to anxious arousal and depression, would be associated with extended processing. To address these hypotheses, zero-order correlations were examined. Depression was not correlated with slowwave amplitude. Anxious arousal was correlated with slow-wave amplitude to positive and negative stimuli over the left hemisphere. Neither emotion Repair or Attention to emotion was correlated with slow-wave. However, Clarity of emotion was correlated with slow wave to negative stimuli over the left hemisphere $(r=.30, p=.02)$. To identify the shared and distinct contributions of anxious arousal and Clarity of emotion to slow wave to negative stimuli, a regression was done with anxious arousal and Clarity of emotion predicting slow wave over the left posterior region. This model accounted for $11 \%$ of the variance for negative stimuli recorded over the left hemisphere $(p=.03)$. Each predictor added variance when added first, clarity of emotion: $\Delta R^{2}=.09, t=2.5$, $p=.02$; anxious arousal: $\Delta R^{2}=.07, t=-2.1, p=.04$, but neither was significant when added last, anxious arousal: $\Delta R^{2}=$ $.016, t=-1.0, p=.31$; Clarity of emotion: $\Delta R^{2}=.04, t=1.6$, $p=.11$. Thus, variance common to anxious arousal and Clarity of emotion accounted for variance in slow-wave amplitude to negative stimuli.

To determine whether this finding was specific to negative stimuli, another series of hierarchical regressions was done. Both slow wave to positive stimuli and slow wave to neutral stimuli were entered in the first step as predictors of slow wave to negative stimuli. Clarity of emotion and anxious arousal were added either second or third. Anxious arousal did not add significant variance with or without Clarity of emotion included. In contrast, clarity of emotion marginally contributed whether anxious arousal was already included, $\Delta R^{2}=.013, t=1.52, p=.13$, or not, $\Delta R^{2}=.018$, $t=1.79, p=.079$.

\section{Discussion}

The present study investigated how PEI, in combination with anxiety and depression, is related to processing of emotional stimuli. It was hypothesized that Attention to emotion and anxious arousal would each be associated with heightened early perception and that Clarity of emotion and anxious apprehension would be associated with increased time to categorize stimuli. Both emotion

Table 4

Regressions Predicting ERP Components

\begin{tabular}{|c|c|c|c|c|c|c|c|c|c|c|c|c|c|}
\hline \multirow[b]{2}{*}{ Dependent variable } & \multirow[b]{2}{*}{ Predictors } & \multicolumn{4}{|c|}{ Positive stimuli } & \multicolumn{4}{|c|}{ Neutral stimuli } & \multicolumn{4}{|c|}{ Negative stimuli } \\
\hline & & $R^{2}$ & $\Delta R^{2}$ & $F, t$ & $p$ & $R^{2}$ & $\Delta R^{2}$ & $F, t$ & $p$ & $R^{2}$ & $\Delta R^{2}$ & $F, t$ & $p$ \\
\hline P200 amplitude: & Full model & .16 & & 5.51 & $<.01$ & .13 & & 4.21 & .02 & .14 & & 4.83 & .01 \\
\hline \multirow[t]{2}{*}{ Left frontal } & Anx arou added last & & .04 & 1.75 & .09 & & .05 & 1.90 & .06 & & .06 & 2.10 & .04 \\
\hline & $\mathrm{AE}$ added last & & .14 & 3.10 & $<.01$ & & .09 & 2.52 & .02 & & .10 & 2.65 & .01 \\
\hline P200 amplitude: & Full model & .14 & & 4.60 & .01 & .14 & & 4.79 & .01 & .13 & & 4.47 & .02 \\
\hline \multirow[t]{2}{*}{ Right frontal } & Anx arou added last & & .06 & 1.98 & .05 & & .07 & 2.22 & .03 & & .10 & 2.55 & .01 \\
\hline & AE added last & & .10 & 2.62 & .01 & & .09 & 2.54 & .01 & & .06 & 2.00 & .05 \\
\hline P300 latency: & Full model & .01 & & .27 & .77 & .03 & & .86 & .43 & .01 & & .32 & .73 \\
\hline \multirow{2}{*}{ Left posterior } & Anx app added last & & .01 & -.73 & .47 & & .01 & -.84 & .41 & & .01 & -.78 & .44 \\
\hline & $\mathrm{CE}$ added last & & .001 & -.27 & .79 & & .01 & .74 & .46 & & .00 & -.02 & .98 \\
\hline P300 latency: & Full model & .04 & & 1.16 & .32 & .09 & & 2.83 & .07 & .12 & & 3.86 & .03 \\
\hline \multirow{2}{*}{ Right posterior } & Anx app added last & & .01 & -.69 & .49 & & .01 & -.82 & .41 & & .05 & -1.86 & .07 \\
\hline & $\mathrm{CE}$ added last & & .02 & 1.13 & .27 & & .06 & 1.93 & .06 & & .03 & 1.49 & .14 \\
\hline
\end{tabular}

Note. $\mathrm{ERP}=$ event-related potential; $\mathrm{Anx}$ arou $=$ anxious arousal; $\mathrm{AE}=$ Attention to emotion; $\mathrm{Anx}$ app $=$ anxious apprehension; $\mathrm{CE}=\mathrm{Clarity}$ of emotion. 
Repair and Attention to emotion, in addition to anxious arousal and depression, would be associated with extended processing, but in different emotion conditions. Overall, results indicated that the Attention to emotion facet of PEI and anxious arousal had similar effects early in the trial, whereas Clarity of emotion and anxious arousal accounted for overlapping variance later in the trial. Clarity of emotion and anxious apprehension shared variance in predicting time to categorize stimuli. These observations indicate that facets of PEI and dimensions of anxiety predict basic emotion stimulus processing and that depression does not.

The hypothesized relationship between anxious arousal and early perception of all stimulus conditions was supported, reflected in results for P200. Surprisingly, Attention to emotion was also associated with P200 in all three emotion conditions. Despite their nonsignificant correlation in this substantial sample, anxious arousal and Attention to emotion may function similarly, leading to vigilance for all upcoming stimuli. This phenomenon is consistent with Clark's (1986) cognitive model of panic, which proposes that an individual prone to panic attacks misinterprets harmless physical sensations as dangerous, thus triggering panic attacks. This lack of inhibition of early affective processing of harmless or neutral stimuli leads to the same reactions to neutral stimuli as to emotional stimuli (Windmann et al., 2002). In the present study, self-reported Attention to emotion combined with anxious arousal was related to early perception of stimuli, regardless of emotional content. This may account for some of inconsistencies in the literature about the relationship between psychopathology and different facets of PEI.

In contrast to nonspecific early attention, emotional content was differentiated as a function of anxiety and PEI further downstream. In combination, anxious apprehension and Clarity of emotion predicted P300 latency to negative stimuli measured over the right hemisphere. Associations between anxious apprehension and Clarity of emotion with P300 latency were predicted, but it was unexpected that anxious apprehension would be associated with shorter, rather than delayed P300 latency for negative stimuli. However, this result is consistent with evidence that anxiety is associated with facilitated processing of fear-relevant targets compared to processing of stimuli that are not feared (Öhman et al., 2001). For example, fear of seeing a spider primes the individual to react quickly, with little effort, when presented with the stimulus because related action plans about spiders are already present (Lang, 1979). Perhaps individuals with high anxious apprehension required less time to evaluate negative stimuli because they were already primed for negative information. These results suggest the hypothesis that low anxious apprehension allows one to spend more time processing negative stimuli, leading to more emotional clarity. This effect was observed over the right hemisphere, not the left. Although a strong statement about this lateralization cannot be made, as the generators of P300 in this study are unknown, the right hemisphere is proficient at interpreting emotional information and achieving a holistic perspective, which may be particularly important for emotional clarity (Heller, 1994).

Later in the processing stream, anxious arousal and Clarity of emotion were associated with opposite patterns of extended processing of stimuli. Anxious arousal was associated with increased (more negative going) slow wave, as predicted, and consistent with Pauli et al. (1997). Clarity of emotion was associated with reduced slow wave. In combination with results reviewed above, slow- wave results suggest that those who perceive themselves to be clear about their emotions require less extended processing because they have categorized the stimuli during the P300 latency window. Anxious arousal, on the other hand, is associated with both early attention to all stimuli and extended processing of emotional stimuli, which is consistent with evidence that anxious arousal (panic disorder) is associated with generalized attention to all types of stimuli (Windmann et al., 2002) and later delayed disengagement from emotional stimuli (e.g., Fox et al., 2001). In retrospect, Emotion repair may not be associated with slow wave, as originally predicted, because Emotion repair involves reinterpreting emotional events that would occur much later in the processing stream. It is possible that Emotion repair is associated with later processing in paradigms involving longer stimulus processing time or ITIs than was the case in the present study.

The prediction that depression would be associated with slow wave was not supported. This result may be due to present task demands. Studies that observed slow-wave differences in depression used working memory tasks. Individuals who are depressed are hypothesized to lack approach motivation (Robinson, Meier, Tamir, Wilkowski, \& Ode, 2009) and thus may not engage in elaborative processing unless required by task demands (e.g., remembering the stimuli for an upcoming recall or recognition session).

Slow wave in this study did mimic slow wave observed in memory tasks in another way. Activity near the slow-wave window used in this study is sometimes referred to as late posterior negativity (LPN), which reflects search and retrieval of source information that was initially engaged when items were processed during the study phase of a memory paradigm (Herron, 2007). LPN in an emotional memory task was not influenced by emotional content (Koenig \& Mecklinger, 2008), which is consistent with present data in which slow wave did not differ by emotion condition.

Given this interpretation, it is interesting that anxious arousal predicted both P200 and slow wave. If activity in this later slowwave window reflects evaluation of information that was engaged when items were processed early on, it makes sense that anxious arousal was associated with both early (P200) and later (slow wave) processes. The association of anxious arousal with both enhanced P200 and slow wave is consistent with evidence that anxious individuals direct attention toward threat during early stages of processing and have difficulty disengaging during later stages (e.g., Fox et al., 2001; Yiend \& Mathews, 2001). This pattern was associated with all stimuli during the P200 window and then was specific to emotional stimuli (both positive and negative) during the slow-wave window, indicating increased focus on arousing stimuli. However, this pattern of potential delayed disengagement was not specific to negative stimuli. This discrepancy could be due to the fact that previous studies did not separate anxious apprehension and anxious arousal and may have oversampled aspects of anxiety that are biased to process negative stimuli. For instance, in the present study P300 latency results were specific to anxious apprehension and negative stimuli. In contrast, anxious arousal was associated with P200 to all stimulus conditions and slow wave to emotional conditions. Because both types of anxiety are associated with reaction to negative stimuli, it is possible that previous studies confounded these two anxiety types, which overemphasized reaction to negative stimuli. 
In addition to addressing primary hypotheses, overall differences between emotion conditions were analyzed to ensure that the paradigm was successful in manipulating emotion effects. Indeed, both P100 and P300 amplitude were larger to positive and negative stimuli than to neutral stimuli. The marginal significance level of both findings can be considered sufficient, as they warrant a one-tailed test in the context of extensive past reports of larger P100 and P300 to emotional than neutral stimuli (e.g., Fox et al., 2008; Herbert, Kissler, Junghöfer, Peyk, \& Rockstroh, 2006; Holmes et al., 2008; X. Li et al., 2005; Schupp, Junghöfer, Weike, \& Hamm, 2003). In present data, positive stimuli prompted later P300 latency than did neutral or negative stimuli. Reports of P300 latency are scarce in the emotion Stroop literature, but Metzger and Orr (1997) reported a trend for later P300 latency to trauma-related words in patients with PTSD. This finding indicates delayed evaluation of such words. Given that this study's sample was nonclinical and that P300 amplitude effects indicated increased attentional allocation to both positive and negative stimuli, perhaps positive stimuli were more salient for this sample, which is consistent with other evidence that healthy controls preferentially process positive information (Engels et al., 2007; Herbert et al., 2006; Herrington et al., 2005).

The present study demonstrated how anxiety and PEI together affect processing of emotional and neutral stimuli. Anxious apprehension was associated with decreased processing time for negative stimuli, which may lead to worse emotional clarity. In contrast, high anxious arousal and Attention to emotion were associated with increased early processing of stimuli. Anxious arousal was also associated with delayed disengagement from stimuli. This pattern of behavior is consistent with clinical observation. Individuals with generalized anxiety disorder tend to worry about future events (e.g., Dugas et al., 1998), whereas individuals who experience anxious arousal symptoms tend to react to the perceived threat and then have difficulty disengaging from it (Fox et al., 2001; Yiend \& Mathews, 2001). Further research in this area may lead to therapies that focus on minimization of anxiety to help individuals regulate their emotions more effectively.

\section{References}

Bar-Haim, Y., Lamy, D., Pergamin, L., Bakermans-Kranenburg, M. J., \& van IJzendoorn, M. H. (2007). Threat-related attentional bias in anxious and nonanxious individuals: A meta-analytic study. Psychological Bulletin, 133, 1-24.

Berenbaum, H., \& Oltmanns, T. F. (1992). Emotional experience and expression in schizophrenia and depression. Journal of Abnormal Psychology, 101, 37-44.

Berenbaum, H., Raghavan, C., Le, H.-N., Vernon, L. L., \& Gomez, J. J. (2003). A taxonomy of emotional disturbances. Clinical Psychology Science and Practice, 10, 206-226.

Berg, P., \& Scherg, M. (1994). A multiple source approach to the correction of eye artifacts. Electroencephalography \& Clinical Neurophysiology, 90, 229-241.

Bradley, M. M., \& Lang, P. J. (1999). Affective norms for English words $(A N E W)$. Gainesville: University of Florida, NIMH Center for the Study of Emotion and Attention.

Bredemeier, K., Berenbaum, H., Boden, M. T., \& Thompson, R. J. (2007, October). Emotional styles, anhedonic depression, and worry. Poster presented at the annual meeting of the Society for Research on Psychopathology, IA.
Clark, D. M. (1986). A cognitive approach to panic. Behaviour Research and Therapy, 24, 461-470.

Coffey, E., Berenbaum, H., \& Kerns, J. (2003). The dimensions of emotional intelligence, alexithymia, and mood awareness: Associations with personality and performance on an emotional Stroop task. Cognition \& Emotion, 17, 671-679.

Compton, R. J., Heller, W., Banich, M. T., Palmieri, P. A., \& Miller, G. A. (2000). Responding to threat: Hemispheric asymmetries and interhemispheric division of input. Neuropsychology, 14, 254-264.

Cook, E. W., \& Miller, G. A. (1992). Digital filtering: Background and tutorial for psychophysiologists. Psychophysiology, 29, 350-367.

Davidson, R., Pizzagalli, D., Nitschke, J., \& Putnam, K. (2002). Depression: Perspectives from affective neuroscience. Annual Review of Psychology, 53, 545-574.

Deveney, C. M., \& Deldin, P. J. (2004). Memory of faces: A slow wave ERP study of major depression. Emotion, 4, 295-304.

Donchin, E., \& Coles, M. G. H. (1988). Is the P300 component a manifestation of cognitive updating? Behavioral and Brain Sciences, 11 357-427.

Drake, M. E., Pakalnis, A., Phillips, B., Pamadan, H., \& Hietter, S. A. (1991). Auditory evoked potentials in anxiety disorder. Clinical Electroencephalography, 22, 97-101.

Dugas, M. J., Freeston, M. H., Ladouceur, R., Rheaume, J., Provencher, M., \& Boisvert, J. M. (1998). Worry themes in primary GAD, secondary GAD, and other anxiety disorders. Journal of Anxiety Disorders, 12, 253-261.

Dunn, B. D., Dalgleish, T., Lawrence, A. D., Cusack, R., \& Ogilvie, A. D. (2004). Categorical and dimensional reports of experienced affect to emotion-inducing pictures in depression. Journal of Abnormal Psychology, 113, 654-660.

Egloff, B., \& Hock, M. (2003). Assessing attention allocation toward threat-related stimuli: A comparison of the emotional Stroop task and the attentional probe task. Personality and Individual Differences, 35, 475 483.

Engels, A. S., Heller, W., Mohanty, A., Herrington, J. D., Banich, M. T., Webb, A. G., \& Miller, G. A. (2007). Specificity of regional brain activity in anxiety types during emotion processing. Psychophysiology, 44, 352-363.

Extremera, N., Durán, A., \& Rey, L. (2007). Perceived emotional intelligence and dispositional optimism-pessimism: Analyzing their role in predicting psychological adjustment among adolescents. Personality and Individual Differences, 42, 1069-1079.

Fernandes, L., \& Miller, G. A. (1995). Compromised performance and abnormal psychophysiology associated with the Wisconsin scales of psychosis proneness. In G. A. Miller (Ed.), The behavioral high-risk paradigm in psychopathology (pp. 47-87). New York, NY: Springer.

Fernández-Berrocal, P., Alcaide, R., Extremera, N., \& Pizarro, D. (2006). The role of emotional intelligence in anxiety and depression among adolescents. Individual Differences Research, 4, 16-27.

First, M. B., Spitzer, R. L., Gibbon, M., \& Williams, J. B. W. (1997). Structured clinical interview for DSM-IV Axis I disorders-Non-patient edition (SCID-I/NP, Version 2.0, Rev. ed.). New York, NY: Biometrics Research Department.

Fox, E. (1993). Attentional bias in anxiety: Selective or not? Behaviour Research and Therapy, 31, 487-493.

Fox, E., Derakshan, N., \& Shoker, L. (2008). Trait anxiety modulates the electrophysiological indices of rapid spatial orienting towards angry faces. NeuroReport, 19, 259-263.

Fox, E., Russo, R., Bowles, R., \& Dutton, K. (2001). Do threatening stimuli draw or hold visual attention in subclinical anxiety? Journal of Experimental Psychology: General, 130, 681-700.

Gohm, C. L., Corser, G. C., \& Dalsky, D. J. (2005). Emotional intelligence under stress: Useful, unnecessary, or irrelevant? Personality and Individual Differences, 39, 1017-1028. 
Gotlib, I. H., \& Cane, D. B. (1987). Construct accessibility and clinical depression: A longitudinal investigation. Journal of Abnormal Psychology, 96, 199-204.

Hanatani, T., Sumi, N., Taguchi, S., Fujimoto, O., Nan-no, H., \& Takeda, M. (2005). Event-related potentials in panic disorder and generalized anxiety disorder. Psychiatry and Clinical Neurosciences, 59, 83-88.

Heller, W. (1994). Cognitive and emotional organization of the brain: Influences on the creation and perception of art. In D. Zaidel (Ed.), Neuropsychology (pp. 271-292). San Diego, CA: Academic Press.

Heller, W., \& Nitschke, J. (1997). Regional brain activity in emotion: A framework for understanding cognition in depression. Cognition \& Emotion, 11, 637-661.

Heller, W., Nitschke, J. B., Etienne, M. A., \& Miller, G. A. (1997). Patterns of regional brain activity differentiate types of anxiety. Journal of Abnormal Psychology, 106, 376-385.

Herbert, C., Kissler, J., Junghöfer, M., Peyk, P., \& Rockstroh, B. (2006). Processing of emotional adjectives: Evidence from startle EMG and ERPs. Psychophysiology, 43, 197-206.

Herrington, J. D., Heller, W., Mohanty, A., Engels, A., Banich, M. T., Webb, A. W., \& Miller, G. A. (in press). Localization of asymmetric brain function in emotion and depression. Psychophysiology.

Herrington, J. D., Mohanty, A., Koven, N. S., Fisher, J. E., Stewart, J. L., Banich, M. T., ... Heller, W. (2005). Emotion-modulated performance and activity in left dorsolateral prefrontal cortex. Emotion, 5, 200-207.

Herron, J. E. (2007). Decomposition of the ERP late posterior negativity: Effects of retrieval and response fluency. Psychophysiology, 44, 233244

Holmes, A., Nielsen, M. K., \& Green, S. (2008). Effects of anxiety on the processing of fearful and happy faces: An event-related potential study. Biological Psychology, 77, 159-173.

Ilan, A. B., \& Polich, J. (1999). P300 and response time from a manual Stroop task. Clinical Neurophysiology, 110, 367-373.

Junghöfer, M., Bradley, M. M., Elbert, T. R., \& Lang, P. J. (2001). Fleeting images: A new look at early emotion discrimination. Psychophysiology, 38, 175-178.

Keil, A., Bradley, M. M., Hauk, O., Rockstroh, B., Elbert, T., \& Lang, P. (2002). Large-scale neural correlates of affective picture processing. Psychophysiology, 39, 641-649.

Keller, J., Nitschke, J. B., Bhargava, T., Deldin, P. J., Gergen, J. A., Miller, G. A., ... Heller, W. (2000). Neuropsychological differentiation of depression and anxiety. Journal of Abnormal Psychology, 109, 3-10.

Koenig, S., \& Mecklinger, A. (2008). Electrophysiological correlates of encoding and retrieving emotional events. Emotion, 8, 162-173.

Lang, P. (1979). A Bio-informational theory of emotional imagery. Psychophysiology, 16, 495-512.

Li, W., Zinbarg, R. E., \& Paller, K. A. (2007). Trait anxiety modulates supraliminal and subliminal threat: Brain potential evidence for early and late processing influences. Cognitive, Affective \& Behavioral Neuroscience, 7, 25-36.

Li, X., Li, X., \& Luo, Y.-J. (2005). Anxiety and attentional bias for threat: An event-related potential study. NeuroReport, 16, 1501-1505.

Lim, S. L., \& Kim, J. H. (2005). Cognitive processing of emotional information in depression, panic, and somatoform disorder. Journal of Abnormal Psychology, 114, 50-61.

Luck, S. J. (2005). An Introduction to the Event-related potential technique. Cambridge, MA: MIT Press.

Mathews, A., \& MacLeod, C. (1985). Selective processing of threat cues in anxiety states. Behaviour Research and Therapy, 23, 563-569.

Mayer, J. D., \& Salovey, P. (1997). What is emotional intelligence? In P. Salovey \& D. Sluyter (Eds.), Emotional development and emotional intelligence: Implications for educators (pp. 3-31). New York: Basic Books.

McCabe, S. B., \& Gotlib, I. H. (1995). Selective attention and clinical depression: Performance on a deployment-of-attention task. Journal of Abnormal Psychology, 104, 241-245.

McNeely, H. E., Lau, M. A., Christensen, B. K., \& Alain, C. (2008). Neurophysiological evidence of cognitive inhibition anomalies in persons with major depressive disorder. Clinical Neurophysiology, 119, $1578-1589$.

Metzger, L. J., \& Orr, S. P. (1997). Seeking the source of emotional Stroop interference effects in PTSD: A study of P3s to traumatic words. Integrative Physiological \& Behavioral Science, 32, 43-51.

Meyer, T. J., Miller, M. L., Metzger, R. L., \& Borkovec, T. D. (1990). Development and validation of the Penn State Worry Questionnaire. Behavior Research and Therapy, 28, 487-495.

Molina, S., \& Borkovec, T. D. (1994). The Penn State Worry Questionnaire: Psychometric properties and associated characteristics. In G. C. L. Davey \& F. Tallis (Eds.), Worrying: Perspectives on theory, assessment and treatment (pp. 265-283). Chichester, England: Wiley.

Nitschke, J. B., Heller, W., Imig, J. C., McDonald, R. P., \& Miller, G. A. (2001). Distinguishing dimensions of anxiety and depression. Cognitive Therapy and Research, 25, 1-22.

Nitschke, J. B., Heller, W., Palmieri, P. A., \& Miller, G. A. (1999) Contrasting patterns of brain activity in anxious apprehension and anxious arousal. Psychophysiology, 36, 628-637.

Nitschke, J. B., Miller, G. A., \& Cook, E. W., III. (1998). Digital filtering in EEG/ERP analysis: Some technical and empirical comparisons. Behavior Research Methods, Instruments \& Computers, 30, 54-67.

Öhman, A., Flykt, A., \& Esteves, F. (2001). Emotion drives attention: Detecting the snake in the grass. Journal of Experimental Psychology General, 130, 466-478.

Oldfield, R. C. (1971). The assessment and analysis of handedness: The Edinburgh Inventory. Neuropsychologia, 9, 97-113.

Pauli, P., Amrhein, C., Mühlberger, A., Dengler, W., \& Wiedemann, G. (2005). Electrocortical evidence for an early abnormal processing of panic-related words in panic disorder patients. International Journal of Psychophysiology, 57, 33-41.

Pauli, P., Dengler, W., Wiedemann, G., Montoya, P., Flor, H., Birbaumer, N., \& Buchkremer, G. (1997). Behavioral and neurophysiological evi dence for altered processing of anxiety-related words in panic disorder. Journal of Abnormal Psychology, 106, 213-220.

Pérez-Edgar, K., \& Fox, N. A. (2003). Individual differences in children's performance during an emotional Stroop task: A behavioral and electrophysiological study. Brain and Cognition, 52, 33-51.

Perrin, F., Pernier, J., Bertrand, O., \& Echallier, J. F. (1989). Spherical splines for scalp potential and current density mapping. Electroencephalography and Clinical Neurophysiology, 72, 184-187.

Pflugshaupt, T., Mosimann, U. P., von Wartburg, R., Schmitt, W., Nyffeler, T., \& Muri, R. M. (2005). Hypervigilance-avoidance pattern in spider phobia. Journal of Anxiety Disorders, 19, 105-116.

Pourtois, G., Grandjean, D., Sander, D., \& Vuilleumier, P. (2004). Electrophysiological Correlates of Rapid Spatial Orienting Towards Fearful Faces. Cerebral Cortex, 14, 619-633.

Ramos, N. S., Fernandez-Berrocal, P., \& Extremera, N. (2007). Perceived emotional intelligence facilitates cognitive-emotional processes of adaptation to an acute stressor. Cognition \& Emotion, 21, 758-772.

Rinck, M., Reinecke, A., Ellwart, T., Heuer, K., \& Becker, E. S. (2005). Speeded detection and increased distraction in fear of spiders: Evidence from eye movements. Journal of Abnormal Psychology, 114, 235-248.

Robinson, M. D., Meier, B. P., Tamir, M., Wilkowski, B. M., \& Ode, S. (2009). Behavioral facilitation: A cognitive model of individual differences in approach motivation. Emotion, 9, 70-82.

Rohner, J.-C. (2002). The time-course of visual threat processing: High trait anxious individuals eventually avert their gaze from angry faces. Cognition \& Emotion, 16, 837-844.

Rude, S., \& McCarthy, C. (2003). Brief report. Cognition \& Emotion, 17, 799. 
Salovey, P., \& Mayer, J. D. (1990). Emotional intelligence. Imagination, Cognition, and Personality, 9, 185-211.

Salovey, P., Mayer, J. D., Goldman, S. L., Turvey, C., \& Palfai, T. P. (1995). Emotional attention, clarity, and repair: Exploring emotional intelligence using the trait meta-mood scale. In J. Pennebaker (Ed.), Emotion, disclosure, \& health (pp. 125-154). Washington, DC: American Psychological Association.

Salovey, P., Woolery, A., \& Mayer, J. D. (2001). Emotional intelligence: Conceptualization and measurement. In G. J. O. Fletcher \& M. S. Clark (Eds.), Blackwell handbook of social psychology: Interpersonal processes (pp. 279-307). Malden, MA: Blackwell.

Salovey, P., Woolery, A., Stroud, L., \& Epel, E. (2002). Perceived emotional intelligence, stress reactivity and symptom reports: Furthers explorations using the Trait Meta-Mood Scale. Psychology and Health, 77, 611-627.

Salters-Pedneault, K., Roemer, L., Tull, M. T., Rucker, L., \& Mennin, D. S. (2006). Evidence of broad deficits in emotion regulation associated with chronic worry and generalized anxiety disorder. Cognitive Therapy and Research, 30, 469-480.

Sass, S. M., Heller, W., Stewart, J. L., Silton, R. L., Edgar, C., Fisher, J., \& Miller, G. (in press). Time course of attentional bias to threat in anxiety: Emotion and gender specificity. Psychophysiology.

Schupp, H. T., Junghöfer, M., Weike, A. I., \& Hamm, A. O. (2003). Emotional facilitation of sensory processing in the visual cortex. Psychological Science, 14, 7-13.

Schupp, H. T., Junghöfer, M., Weike, A. I., \& Hamm, A. O. (2004). The selective processing of briefly presented affective pictures: An ERP analysis. Psychophysiology, 41, 441-449.

Shestyuk, A. Y., Deldin, P. J., Brand, J. E., \& Deveney, C. M. (2005). Reduced sustained brain activity during processing of positive emotional stimuli in major depression. Biological Psychiatry, 57, 1089-1096.

Spielberger, C. D., Gorsuch, R. L., \& Lushene, R. E. (1970). Manual for the State-Trait Anxiety Inventory, Palo Alto, CA: Consulting Psychologists Press.

Thomas, S. J., Johnstone, S. J., \& Gonsalvez, C. J. (2007). Event-related potentials during an emotional Stroop task. International Journal of Psychophysiology, 63, 221-231.

Toglia, M. P., \& Battig, W. F. (1978). Handbook of semantic word norms. Hillsdale, NJ: Erlbaum.

Tull, M. T., \& Roemer, L. (2007). Emotion regulation difficulties associ- ated with the experience of uncued panic attacks: Evidence of experiential avoidance, emotional non-acceptance, and decreased emotional clarity. Behavior Therapy, 38, 378-391.

Turan, T., Esel, E., Karaaslan, F., Basturk, M., Oguz, A., \& Yabanoglu, I. (2002). Auditory event-related potentials in panic and generalized anxiety disorders. Progress in Neuro-Psychopharmacology \& Biological Psychiatry, 26, 123-126.

van Hooff, J. C., Dietz, K. C., Sharma, D., \& Bowman, H. (2008). Neural correlates of intrusion of emotion words in a modified Stroop task. International Journal of Psychophysiology, 67, 23-34.

Watson, D., Clark, L. A., Weber, K., Assenheimer, J. S., Strauss, M. E., \& McCormick, R. A. (1995). Testing a tripartite model: II. Exploring the symptom structure of anxiety and depression in student, adult, and patient samples. Journal of Abnormal Psychology, 104, 15-25.

Watson, D., Weber, K., Assenmeimer, J. S., Clark, L. A., Strauss, M. E., \& McCormick, R. A. (1995). Testing a tripartite model: I. Evaluating the convergent and discriminant validity of anxiety and depression symptom scales. Journal of Abnormal Psychology, 104, 3-14.

West, R., \& Alain, C. (2000). Effects of task context and fluctuations of attention on neural activity supporting performance of the Stroop task. Brain Research, 873, 102-111.

Williams, J. M. G., Mathews, A., \& MacLeod, C. (1996). The emotional Stroop task and psychopathology. Psychological Bulletin, 120, 3-24.

Williams, J. M. G., \& Nulty, D. D. (1986). Construct accessibility, depression and the emotional Stroop task: Transient mood or stable structure? Personality and Individual Differences, 7, 85-491.

Windmann, S., Sakhavat, Z., \& Kutas, M. (2002). Electrophysiological evidence reveals affective evaluation deficits early in stimulus processing in patients with panic disorder. Journal of Abnormal Psychology, 111, 357-369.

Yee, C. M., \& Miller, G. A. (1994). A dual-task analysis of resource allocation in dysthymia and anhedonia. Journal of Abnormal Psychology, 103, 625-636.

Yiend, J., \& Mathews, A. (2001). Anxiety and attention to threatening pictures. Quarterly Journal of Experimental Psychology: Human Experimental Psychology, 3, 665-681.

Received December 29, 2008

Revision received November 24, 2009

Accepted December 8, 2009

\section{E-Mail Notification of Your Latest Issue Online!}

Would you like to know when the next issue of your favorite APA journal will be available online? This service is now available to you. Sign up at http://notify.apa.org/ and you will be notified by e-mail when issues of interest to you become available! 